$11-1-2008$

\title{
The Value of Intraoperative Examination of Axillary Sentinel Nodes in Carcinoma of the Breast.
}

\author{
Gordon F. Schwartz \\ Thomas Jefferson University \\ Lauren S. Krill \\ Thomas Jefferson University \\ Juan P. Palazzo \\ Thomas Jefferson University \\ Abhijit Dasgupta \\ Thomas Jefferson University
}

Follow this and additional works at: https://jdc.jefferson.edu/pacbfp

Part of the Medical Cell Biology Commons

Let us know how access to this document benefits you

\section{Recommended Citation}

Schwartz, Gordon F.; Krill, Lauren S.; Palazzo, Juan P.; and Dasgupta, Abhijit, "The Value of Intraoperative Examination of Axillary Sentinel Nodes in Carcinoma of the Breast." (2008). Department of Pathology, Anatomy, and Cell Biology Faculty Papers. Paper 22.

https://jdc.jefferson.edu/pacbfp/22

This Article is brought to you for free and open access by the Jefferson Digital Commons. The Jefferson Digital Commons is a service of Thomas Jefferson University's Center for Teaching and Learning (CTL). The Commons is a showcase for Jefferson books and journals, peer-reviewed scholarly publications, unique historical collections from the University archives, and teaching tools. The Jefferson Digital Commons allows researchers and interested readers anywhere in the world to learn about and keep up to date with Jefferson scholarship. This article has been accepted for inclusion in Department of Pathology, Anatomy, and Cell Biology Faculty Papers by an authorized administrator of the Jefferson Digital Commons. For more information, please contact: JeffersonDigitalCommons@jefferson.edu. 
As submitted to Journal of the American College of Surgeons and later published as: Volume 207, Issue 5, November 2008, Pages 758-762

DOI: 10.1016/j.jamcollsurg.2008.06.341

The Value of Intraoperative Examination of Axillary Sentinel Nodes in Carcinoma of the Breast.

Schwartz GF, Krill LS, Palazzo JA, Dasgupta A. Jefferson Medical College, Philadelphia, PA.

Abstract

Axillary sentinel lymph node biopsy (SLNB) has become the standard of care for T1-2, $\mathrm{N}-0, \mathrm{M}-0$ carcinoma of the breast. However, the accuracy of frozen section in the intraoperative examination of sentinel nodes (SN) remains controversial. The senior author has championed the use of the intraoperative examination of $\mathrm{SN}$ by frozen section examination from the inception of its use, and we present our experience with frozen section examination of $\mathrm{SN}$, confirming that this technique is both practical and highly accurate.

Materials \& Methods:

Between 2000 and 2007, 236 SLNB procedures were performed that were read as "frozen section negative." SN were identified by $1 \%$ lymphazurin blue dye only. The identification of SN in these 236 women was $100 \%$. Each SN specimen was prosected by the senior author; a dedicated surgical pathology technician prepared the frozen sections. Nodes were dissected from the specimen individually and cut at 2.5 to $3.0 \mathrm{~mm}$. intervals. Each of these sections was then cut at three levels. The frozen sections were read by the attending pathologist assigned to frozen sections for that day, not by a Page 1 of 14 
dedicated breast pathologist. During the period of the study, 14 different attending pathologists read the slides, with 6 pathologists each reading more than 20 cases. Others read from 1 to 14 cases.

Results:

In this group of 236 cases, 11 patients had positive nodes on subsequent examination of the H\&E slides; thus, the false negative rate of intraoperative frozen section was $4.7 \%$, i.e., the frozen section was read as negative but the paraffin sections were positive for metastasis. Therefore, the sensitivity of the negative frozen section was $>95 \%$. Nine of the 11 false positives were micrometastases, less than $2 \mathrm{~mm}$ diameter, one was considered a macrometastasis, with two areas in one node measuring 2.0 and $2.1 \mathrm{~mm}$ each, and one was a sub-micrometastasis. The following variables were compared for significance: Pathologist, nuclear grade, histologic grade, margins, lymphovascular invasion, tumor type (ductal vs lobular), ER \& PR values. The only significant variables were lymphovascular invasion $(\mathrm{p}=.019)$ and presence of in situ ductal carcinoma $(p=.001)$. Only one of the false negatives was a purely lobular carcinoma $(1 / 11)$.

\section{Discussion:}

Our data confirm the high accuracy of intraoperative examination of $\mathrm{SN},>95 \%$, even without a dedicated breast pathologist reviewing the sections. The missed metastases are likely to be micrometastases, and the likelihood of missing a macrometastasis is $<1 \%$. In patients with large primary tumors, presence of in situ ductal carcinoma or if lymphovascular invasion is associated with the tumor, special care should be taken to review these cases more thoroughly since these characteristics of tumors seem to make them more likely than others to have micrometastases to the axillary nodes overlooked.

Page 2 of 14 
Introduction

Axillary sentinel lymph node biopsy (SLNB) has become the standard of care for T1-2, $\mathrm{N}-0, \mathrm{M}-0$ carcinoma of the breast. ${ }^{1}$ A vexing question that remains controversial is the value of the intraoperative examination of sentinel nodes (SN) by frozen section. Frozen examination of $\mathrm{SN}$ has been reported to miss up to $40 \%$ of metastasis later confirmed by examination of the permanent sections by hematoxylin and eosin staining. ${ }^{2},{ }^{3,4}$, If not performed or if inaccurately performed, the patient has been traditionally committed to a second procedure, i.e., completion axillary dissection of levels I and II if the node(s) examined prove to harbor metastasis. A second procedure increases the costs of care significantly, may be accompanied by additional morbidity since a second operation is required, and has a negative emotional impact on the patient.

The purpose of this study was to determine the negative predictive value of frozen section performed during the intraoperative examination of sentinel nodes in breast cancer patients. In this context, a "false-negative" SLNB implies the finding of metastasis in the formalin-fixed, hematoxylin and eosin (H\&E) stained sentinel nodes after a negative intraoperative frozen section examination. The "negative predictive value" is defined as the number of true negatives divided by the number of false negatives plus the true negatives. Immunohistochemical positivity (cytokeratin staining) only without $\mathrm{H} \& \mathrm{E}$ positivity, even on additional slide review, was not considered a false negative. Additionally, another aim of this study was to identify characteristics of the primary lesion that might indicate patients who are at a higher risk for a false-negative SLNB.

\section{Patients and Methods}

Patients with carcinoma of the breast who underwent sentinel lymph node biopsy at Thomas Jefferson University Hospital, performed by the senior author between January 1, 2000 and December 31, 2006, were identified through a database search and retrospective chart review approved by the Institutional Review Board. Of these cases, 236 Page 3 of 14 
SLNB specimens were read as "frozen section negative." Patient age, date of the procedure, tumor type, tumor size, presence of in situ carcinoma component, nuclear grade, histologic grade, lymphovascular invasion, margin status, ER/PR receptor status, frozen section results, paraffin section results, and immunuhistochemical results were obtained by reviewing pathology and operative reports.

The procedure was executed similarly in each patient. Sentinel nodes were identified by the injection of 3-4 $\mathrm{mL}$ of $1 \%$ isosulfan blue dye into the breast in an intradermal and peritumoral fashion, with one ML always being delivered intradermally and the remainder injected into the breast parenchyma between the tumor site and the lower part of the hair-bearing area of the axilla. The breast was then massaged for four minutes. Longer massage times, up to seven minutes, were used if the patient was obese with very pendulous breasts or the tumor site was far from the intended incision site.

Radioactive tracer was not used in any of these cases. Early in the senior surgeon's experience with SLNB, the experience with blue dye was so positive that radioisotope was abandoned in favor of blue dye only. Our experience with blue dye only has continued to be excellent and the time from incision to retrieval of blue-stained nodes so short that we have continued to use blue dye only, Parenthetically, when isosulfan blue was in short supply, we temporarily used methylene blue dye, but returned to isosulfan blue when the latter again became readily available.

The axillary incision was made in the lower third of the hair-bearing region of the axilla, in the skin lines, behind the edge of the pectoralis muscle and ending in front of the latissimus dorsi muscle. Incision length varied from $2.0 \mathrm{~cm}$ up to $5.0 \mathrm{~cm}$ in length depending upon patient size. One or more blue stained lymphatics leading to the SN could often be identified coming from the breast side of the skin flap, and these could be followed to the SN. If the lymphatics could not be seen superficially, once the first layer of axillary fascia was divided, the blue stained node package could be identified more deeply located. The blue stained package of nodes was excised and sent to pathology Page 4 of 14 
for frozen section examination. Any adjacent non-blue stained nodes that were firm enough to be considered suspicious were also removed. The usual time between incision and excision of the node package was 6-10 minutes.

The senior surgeon (GFS) prosected these node packages personally with the surgical pathology staff in each case, to identify, orient and cut the nodes for frozen section analysis. Each node was oriented along it longitudinal axis, and cut at about 2-3 mm intervals, trying to keep the capsule of each node oriented at the edge of each section. Each of these specimens was then cut at three levels for frozen section examination using hematoxylin and eosin staining. One of two especially trained histology technicians prepared all of the frozen sections. After the frozen sections were performed, the remaining tissue was fixed in formalin and embedded in paraffin; each of these paraffin blocks was cut at three levels, occasionally more, and stained with hematoxylin and eosin for microscopic examination. Immunohistochemical staining with cytokeratin was also employed after fixation, but the results of the cytokeratin staining were not used to influence any therapeutic decisions.

Artifacts can occur in cytokeratin staining that may be mistaken for metastasis, so that sentinel nodes that were positive for cytokeratin only were not considered false negative if re-review of the H\&E sections revealed no evidence of metastasis on light microscopy. Metastases were divided into three categories: submicrometastasis, micrometastasis, and macrometastatsis. "Submicrometastasis" meant individual tumor cells or cell clusters less than $0.2 \mathrm{~mm}$ identified by cytokeratin staining and then confirmed by re-review of the H\&E stained slides. The term "micrometastasis" was used to describe a cohesive cluster of malignant cells $\geq 0.2 \mathrm{~mm}$ up to $2 \mathrm{~mm}$ in diameter seen by light microscopy. A macrometastasis was tumor within a node that measured more than $2 \mathrm{~mm}$ in diameter. Statistical analyses were done using standard statistical methods with the software package $R$ v.2.6.0. ${ }^{6}$

Results

Page 5 of 14 
During the study period, two hundred thirty six women underwent SLNB with intraoperative frozen section examination of their $\mathrm{SN}$ called negative for metastasis. During this same period of time an additional 47 patients underwent SLNB with frozen sections reported as positive for metastatic cancer. Sentinel nodes were identified in each of the 283 women in whom the procedure was attempted. No patient was excluded for failure to identify SN.

The number of blue-stained nodes recovered in each of the "SN frozen section negative" patients ranged from 1 to 9 , with a mean of 3.1. Within this group, 11 of the 236 $(4.7 \%)$ were subsequently found to have metastatic disease on examination of the formalin fixed, H\&E stained, slides. The SN were truly negative in 225 of the 236 cases, so that the negative predictive value and the sensitivity of frozen section were both 95.3\%. No false positives were encountered, i.e., frozen section positive for metastasis, final H\&E slides negative. Specificity was, therefore, $100 \%$.

Of the eleven patients that had positive nodes on subsequent examination of the formalin fixed, H\&E stained slides, nine had micrometastases, less than $2 \mathrm{~mm}$ diameter, one was considered a macrometastasis, with two areas in one node measuring 2.0 and 2.1 $\mathrm{mm}$ each, and one was a sub-micrometastasis. In the latter, cytokeratin stained cells were seen on the IHC staining and later confirmed as tumor cells by review of the H\&E slides. During the period of the study, 14 different attending pathologists read the slides, with 6 pathologists each reading more than 20 cases with the others each reading between 1 and 14 cases.

The ages of the patients ranged from 34 to 83 years with a median of 59 years. There was no age difference between the true negatives and false negatives ( $p$-value $=0.69$ ). There was also no difference in accuracy by the date of surgery, i.e., in the first three years of this study vs the second three years ( $p$-value $=0.84$ ). 
Table 1 demonstrates variables compared for significance including nuclear grade, histologic grade, margins, lymphovascular invasion, primary tumor type (ductal vs lobular, etc.), and presence of DCIS at the tumor site. Tumor size and hormone receptor status were also reviewed but were not significant. The only significant variables were the presence of lymphovascular invasion and presence of in situ ductal carcinoma (DCIS) $(p=.019$ and $p=.001$, respectively). The former is not unexpected, since lymphovascular invasion at the tumor site is considered indicative of the lesion's propensity to metastasize to the lymph nodes. We have no explanation for the significance of DCIS in the primary tumor. Since the slides of the primary tumors were not rereviewed, and the only criterion for DCIS was the pathologists' descriptions of the findings, we do not know the extent of the DCIS in each of these cases.

Only one of the false negatives was a purely lobular carcinoma despite the acknowledged difficulty of seeing these small cells on frozen section slides. Tumor type and other characteristics were not significant, but with only 11 false negatives altogether, there would be a lack of power to see all but very large associations.

The time to perform the frozen section examination ranged between 20 and 30 minutes. While the frozen sections were being prepared and read, the operating team closed the axillary wound, usually completing this part of the procedure a few minutes before the frozen section examination was complete. In the patients with positive nodes, the wound was reopened and a completion dissection of levels I and II was performed. For those with frozen section negative nodes, the procedure was concluded, the wound was dressed and the patient awakened.

Procedures were all performed as out-patient stays, even when the frozen sections detected metastasis and completion axillary dissection performed. The wounds were not drained if $\mathrm{SN}$ were negative, but if a completion dissection was performed, a single 15 French suction catheter was placed through a stab wound in the lower skin flap into the wound along the edge of the latissimus dorsi muscle and connected to a silicone bulb Page 7 of 14 
(Jackson-Pratt ${ }^{\circledR}$ ) reservoir. The drain was removed when the drainage was $<30 \mathrm{~mL} / 24$ hrs.

The final results of the review of the permanent sections were discussed with each patient once the final report had been received. In the 11 patients with the "false negative" results, none underwent subsequent completion dissection of the axilla, and none has (yet) developed axillary metastasis. (Parenthetically, the senior author has all but abandoned completion axillary dissection for patients with micrometastases.)

Discussion

Over the last decade the technique of SLNB has been continually refined. The likelihood of a false-negative biopsy is expected to be than $5 \%$, however false negative is defined. When the final definitive pathology review of the formalin fixed tissue documents sentinel node involvement by tumor, this is not a failure of the technique but a limit of intraoperative frozen section. ${ }^{7}$, The missed metastases are likely to be micrometastases, and the likelihood of missing a macrometastasis is $<1 \%$.

Within the past year, two reports, both from the same institution, have deprecated the value of the intraoperative examination of $\mathrm{SN}$, citing their lack of accuracy of frozen section in identifying axillary metastasis in many patients. ${ }^{4}$, Recognizing their very large numbers that render their statistical calculations meaningful, we do not understand why their sensitivity is so low, compared to ours. Whether this observed difference is related to the difference in the number of patients in the current report and theirs or the combination of an entire institution's surgical experience versus that of a single surgeon is debatable. We have presented our own data to indicate what frozen sections can accomplish rather than what they cannot!

To identify patients at high risk for having false negative frozen sections certain primary tumor characteristics should be considered. In patients with lymphovascular invasion, Page 8 of 14 
special care should be taken when reviewing these cases since this characteristic is more likely than others to be accompanied by metastases to the axillary nodes that may be overlooked during intraoperative frozen section examination. When the initial diagnosis is made by core biopsy, then followed by local excision of the primary tumor and SLNB concurrently, this tumor characteristic may be overlooked until the slides of the entire tumor are reviewed. When, however, the core biopsy does identify lymphovascular invasion adjacent to the tumor, additional attention may be paid to the frozen sections. Although an unexplained but significant finding, we suggest that large tumors accompanied by enough DCIS to warrant a comment by the pathologist should be considered also "at risk," for uncertain reasons.

Several criteria improve the success of frozen section analysis. The tissue sample should be prosected and the frozen sections prepared by an experienced surgical pathologist or technician; however, our data show that it is not necessary to have the slides reviewed by a dedicated breast pathologist. There is a wide range of techniques used in the evaluation of sentinel nodes; in 2002, the Consensus Conference Committee endorsed the recommendations published in the College of American Pathologists Consensus Statement of $1999 .^{7,}$,

Ideally, a low rate of failure to identify SN (sometimes incorrectly also called false negative) should be achieved consistently in order to justify SLNB replacing formal axillary dissection. ${ }^{79}$, This goal is best achieved with training and experience. As noted during the consensus conference in Philadelphia, there are several approaches for learning this technique that have been used by the National Surgical Adjuvant Breast and Bowel Project (NSABP) and American College of Surgeons Oncology Group (ACOSOG)' The panel agreed that at least 20 concomitant axillary dissections should be performed to validate a surgeon's SLNB technique. ${ }^{7}$ Several authorities in the field have also proposed that sentinel node biopsy without further axillary dissection should not be adopted until the surgical team can document the accuracy and demonstrate a failure to identify $\mathrm{SN}$ rate of less than five percent. The authors of the American Society of Clinical OnPage 9 of 14 
cology Guidelines have also suggested that surgeons who perform this procedure should utilize formal courses on the technique, or operate with an experienced mentor, and document their experience, including statistics on successful identification of $\mathrm{SN} .{ }^{10}$

We have shown that it is practical to use frozen section with a low false negative rate. The technique employed is not time-consuming and does not require dedicated breast pathologists to review all of the sections. We, did, however, have the benefit of two specially trained histology technicians, highly skilled in preparing frozen sections. Similar rates of accuracy have been achieved by using what has been called an "exhaustive" technique, examining as many as 60 sections per node. This more labor-intensive method, not commonly feasible in most American hospitals, had a negative predictive value of $95.3 \% .^{2}$ This same institution reported $93.7 \%$ negative predictive value using their "routine" frozen section technique.

Why we have achieved this very high degree of accuracy of intraoperative frozen section examinations of $\mathrm{SN}$ compared to other reported series is not readily apparent. The only variables that we did control compared with reports from other institutions were the surgeon personally prosecting the $\mathrm{SN}$ specimen, separating the nodes from one another, defatting and orienting each node before cutting it into sections, and the dedicated histology technicians preparing and staining the frozen section slides. No innovative or special techniques were employed to prepare the slides for examination. Care was taken to try to orient the node specimens so that areas beneath the node capsule were easily seen in the microscopic fields of the frozen sections.

Frozen section may still fail to detect micrometastases, especially with large tumors, those with accompanying lymphovascular invasion, or those with abundant areas of DCIS associated with the tumor. Although it is unreasonable to expect to eliminate false negative frozen sections altogether, particularly in these mentioned situations, our experience validates the use of intraoperative frozen section examination as an accom 
paniment to SLNB. Our failure to detect metastasis in sentinel nodes was less than $5 \%$, and virtually all of these were micrometastases. For the patients with positive SN who are to undergo completion axillary dissection, it is obviously preferable if that procedure can be performed immediately rather than forcing the patient to return for a second operation. In our own institution, this has not created a scheduling problem in the operating room. We have had enough flexibility in the schedule to allow enough time between cases to permit a completion dissection, when necessary, without creating rebellion or confusion among the surgical and nursing staffs.

Whether the presence of a micrometastasis in a sentinel node justifies completion axillary dissection is another question beyond the scope of this paper, although we believe that it does not. Therefore, at least in our hands and at our institution, intraoperative examination of sentinel nodes by frozen section has avoided a second procedure in all of our patients. Those with documented frozen section positive macrometastases have undergone completion axillary dissection following the sentinel node biopsy, under the same anesthetic, obviating a second procedure. We have accomplished our aim, achieving a high rate of accuracy by frozen section examination and avoiding an additional procedure. We believe that this should be the goal of each surgeon who performs SLNB for carcinoma of the breast. 
TABLE 1 ( $P$ - values are exact $p$-values based on Fisher's exact test)

\begin{tabular}{|c|c|c|c|c|}
\hline Final $\mathrm{H}+\mathrm{E}$ & $\mathbf{N}^{*}$ & Negative & Positive & P-value \\
\hline & & n (\%) & $\mathrm{n}(\%)$ & \\
\hline $\begin{array}{l}\text { In Situ Compo- } \\
\text { nent }\end{array}$ & 218 & & & \\
\hline Absent & & $42(20)$ & $7(70)$ & 0.001 \\
\hline Present & & $166(80)$ & $3(30)$ & \\
\hline Nuclear grade & 217 & & & 0.9 \\
\hline 1 & & $29(14.0)$ & $1(10)$ & \\
\hline 2 & & $107(51.7)$ & $5(50)$ & \\
\hline 3 & & 71 (34.3) & $4(40)$ & \\
\hline Histologic grade & 217 & & & 0.08 \\
\hline 1 & & 35 (16.9) & $1(10)$ & \\
\hline 2 & & $100(48.3)$ & $2(20)$ & \\
\hline 3 & & $72(34.8)$ & $7(70)$ & \\
\hline Margins & 231 & & & 0.72 \\
\hline Negative & & $170(77.3)$ & $8(72.7)$ & \\
\hline Positive & & $50(22.7)$ & $3(27.3)$ & \\
\hline $\begin{array}{l}\text { Vascular inva- } \\
\text { sion }\end{array}$ & 231 & & & 0.019 \\
\hline Negative & & $200(90.9)$ & 7 (63.6) & \\
\hline Positive & & $20(9.1)$ & $4(36.4)$ & \\
\hline Tumor type & 236 & & & \\
\hline Ductal & & $190(84.4)$ & $9(81.8)$ & 0.38 \\
\hline Lobular & & $14(6.2)$ & $1(9.1)$ & \\
\hline $\begin{array}{l}\text { Lobular and Duc- } \\
\text { tal }\end{array}$ & & $9(4.0)$ & $1(9.1)$ & \\
\hline Other & & $12(5.3)$ & $0(0)$ & \\
\hline \multicolumn{5}{|l|}{$\begin{array}{l}\text { Missed metasta- } \\
\text { ses }\end{array}$} \\
\hline macro & & & $1(9.1)$ & \\
\hline micro & & & $9(81.8)$ & \\
\hline submicro & & & $1(9.1)$ & \\
\hline
\end{tabular}


Numbers less

than 236 mean

that variable not

recorded in (236-

n) patients

REFERENCES

1 Veronesi U, Paganellia G, Viale G, et al. A randomized comparison of sentinel node biopsy with routine axillary dissection in breast cancer. The New England Journal of Medicine 2003; 349:546-553.

2 Zurrida S, Mazzarol G, Galimberti V, et al. The problem of the accuracy of intraoperative examination of axillary sentinel nodes in breast cancer. Annals of Surgical Oncology 2001; 8: 817-820.

3 Chao C, Wong SL, Ackermann D, Simpson D, Carter MB, Brown M, Edwards MJ, McMasters KM. Utility of intraoperative frozen section analysis of sentinel lymph nodes in breast cancer. The American Journal of Surgery 2002;182:609-615.

4 Chan SW, LaVigne KA, Port ER, et al: Does the benefit of sentinel node frozen section vary between patients with invasive duct, invasive lobular, and favorable histologic subtypes of breast cancer? Annals of Surgery 2008; 247: 143-149.

5 McLaughlin SA, Ochoa-Frongia LM, Patil SM, et al: Influence of frozen-section analysis of sentinel lymph node and lumpectomy margin status on reoperation rates in patients undergoing breast-conservation therapy. Journal of the American College of Surgeons 2008; 206: 76-82.

Page 13 of 14 
6 R Development Core Team (2007). R: A language and environment for statistical computing. R Foundation for Statistical Computing, Vienna, Austria. ISBN 3900051-07-0, URL http://www.R-project.org.

7 Schwartz GF, Giuliano AE, Veronesi U, et al. Proceedings of the Consensus Conference on the Role of Sentinel Lymph Node Biopsy in Carcinoma of the Breast, April 19-22, 2001, Philadelphia, Pennsylvania. CANCER 2002; 94: 2542-2551.

8 Fitzgibbons PL, Page DL, Weaver D, et al. Prognostic factors in breast cancer. College of American Pathologists Consensus Statement 1999. Archives of Pathology and Laboratory Medicine 1999; 124:966-978.

9 Schwartz GF. Clinical practice guidelines for the use of axillary sentinel lymph node biopsy in carcinoma of the breast: Current update. The Breast Journal 2004; 10 (2):85-88.

10 Lyman GH, Giuliano AE, Somerfield MR, et al. American Society of Clinical Oncology Guideline Recommendations for Sentinel Lymph Node Biopsy in Early-Stage Breast Cancer. Journal of Clinical Oncology 2005; 23:7703-7720.

Page 14 of 14 